Ergod. Th. \& Dynam. Sys. (1987), 7, 175-192

Printed in Great Britain

\title{
Time-like geodesic flows on Lorentz manifolds
}

\author{
GÉRARD G. EMCH AND SUNGPYO HONG \\ Department of Mathematics, University of Florida, Gainesville, FL 32611, USA; \\ Department of Mathematics, The University of Rochester, Rochester, NY 14627, USA
}

(Received 24 January 1985 and revised 22 May 1986)

Abstract. An extension from Riemann to Lorentz manifolds is proved for Anosov's theorem on the hyperbolicity (or exponential sensitivity to initial conditions) of the geodesic flow.

\section{Introduction}

In order to produce an intrinsic proof of the ergodicity of the geodesic flow on a compact Riemann manifold of negative curvature, Anosov (cf. [1], [2]) focused attention on the role played by the asymptotically exponential instability of the flow, thus extending the scope of the Hadamard phenomenon (cf. [8]) of exponential sensitivity to initial conditions: this analysis provided stimulating insights on how chaotic behaviour could be obtained in deterministic, in fact Hamiltonian, systems. Anosov further proved that the hyperbolicity property he isolated is stable.

In classical mechanics, the Galilean principle of relativity allows for a clean separation between time $(\mathbb{P})$ and space $\left(\mathbf{M}_{0}\right)$; Anosov's results are thus directly applicable to this situation: information on space-time trajectories can be lifted readily from the information one has on their spatial projection. The question then arises to how to extend this theory in order to take into account Einstein's (rather than Galileo's) relativity principle. As a first, rather conservative, step in this direction, one may want to consider a Lorentz manifold $\mathbf{M}$ equipped with the structure of a warped product (cf. [16]) of $\mathbb{R}$ (time) with a Riemann manifold $\mathbf{M}_{0}$ (space) of negative curvature. This approach was considered in [15], under some additional assumptions (homogeneity and compactness of $\mathbf{M}_{0}$ ) that lead to rather non-standard cosmologies.

In the present paper, we extend Anosov's theory to a class of manifolds $\mathbf{M}$ that are equipped with Lorentz, rather than Riemann, metrics; in order to avoid violating causality requirements (cf. [10], [16]), these manifolds are not taken to be compact; nor do we assume that M's are warped products. We focus on the study of the dynamical properties of the time-like geodesic flows defined in $\S \S 2,3$. In $\S 4$, we prove, under certain conditions on the time-like sectional (rather than spacial) curvature along time-like geodesics, that these flows satisfy an exponential instability, which we refer to as hyperbolicity, and which we define in $\S 2$. We also discuss in that section (see in particular theorem 2.8) the sense in which hyperbolicity of time-like flows is a stable property; for time-like geodesic flows see remark 4.5 . 
We note that the general conditions under which our results are proved hold in particular for the time-like geodesic flow on complete, simply connected Lorentz manifolds of constant positive curvature; these manifolds are Robertson-Walker space-times (cf. [16], [17], [18]) with $\mathbf{M}$ a warped product of $\mathbb{R}$ and a homogeneous Riemann manifold $\mathbf{M}_{0}$ of positive curvature. This suggests we speculate on the possible application of our type of results in the realm of standard relativistic cosmology; these results indeed mean, in physical terms, that the trajectories of freely falling particles diverge exponentially in time, and thus depend very sensitively on initial conditions, so that the fine structures that might have initially existed in a dust cloud would be very rapidly wiped out unless some deviation from the conditions we have assumed could maintain them in place.

\section{Time-like hyperbolic flows}

Throughout this paper, we say that $(\mathcal{M}, \mathscr{G})$ is a space-time if the following four conditions are satisfied:

(i) $\mathcal{M}$ is a connected, Hausdorff and paracompact smooth manifold of dimension $n \geq 2$;

(ii) $\mathscr{G}$ is a Lorentz metric tensor on $\mathscr{M}$ with signature $(+\cdots,+,-)$;

(iii) $(\mathcal{M}, \mathscr{G})$ is oriented and time-oriented; and

(iv) $(\mathcal{M}, \mathscr{G})$ is equipped with the Levi-Civita connection.

In this section, we assume that $(\mathcal{M}, \mathscr{G})$ is a space-time of dimension $n \geq 3$ and $\xi$ is a complete time-like vector field of class $C^{r}, r \geq 2$, on $\mathcal{M}$, with its complete flow $\left\{\phi_{1} \mid t \in \mathbb{R}\right\}$ such that for some constants $\alpha, \beta$ with $\alpha \geq \beta>0$ and for every $x \in \mathcal{M}$

$$
\beta \leq\left|\mathscr{G}\left(\xi_{x}, \xi_{x}\right)\right| \leq \alpha .
$$

This condition is satisfied naturally by the time-like geodesic flow (cf. $\S 3$ ).

Definition 2.1. For a given $x_{0} \in \mathcal{M}$, the flow line $\left\{x_{t} \equiv \phi_{t} x_{0} \mid t \in \mathbb{R}\right\}$ generated by $\xi$ is said to be hyperbolic if there exists a splitting of $T_{x_{t}} \mathcal{M}$ over the flow line $\left\{x_{t} \mid t \in \mathbb{R}\right\}$ into $T \phi$-invariant subspaces $\mathbf{Z}_{t}^{1} \equiv \mathbf{Z}_{x_{t}}^{1}, \mathbf{V}_{t}^{s} \equiv \mathbf{V}_{x_{t}}^{s}$ and $\mathbf{V}_{t}^{u} \equiv \mathbf{V}_{x_{t}}^{u}$ such that for all $t \in \mathbb{R}$,

$$
T_{x_{1}}, \mathcal{M}=\mathbf{Z}_{t}^{1} \oplus \mathbf{V}_{t}^{s} \oplus \mathbf{V}_{t}^{u}
$$

where the subspaces $Z_{t}^{1}, V_{t}^{s}$ and $V_{t}^{u}$ depend smoothly on $t$, and satisfy the following four conditions:

(i) $\mathbf{Z}_{t}^{1}$ is generated by the time-like vector $\xi\left(x_{t}\right)$;

(ii) $\operatorname{dim} \mathbf{V}_{t}^{s}=k \neq 0$ and $\operatorname{dim} \mathbf{V}_{t}^{u}=l \neq 0$;

(iii) $\mathbf{V}_{t}^{s} \oplus \mathbf{V}_{t}^{u}$ is space-like; and

(iv) $\mathbf{V}_{t}^{s}$ (resp. $\mathbf{V}_{t}^{u}$ ) contracts (resp. expands) exponentially: i.e. there are constants $a \geq 1$ and $b, b^{\prime}>0$ (possibly depending on $x_{0}$ ) such that for all $t>0$ and $\zeta \in \mathbf{V}_{t}^{s}$ (resp. $\eta \in \mathbf{V}_{t}^{u}$ )

$$
\begin{gathered}
a^{-1}\|\zeta\| e^{-b^{\prime} t} \leq\left\|T \phi_{t} \zeta\right\| \leq a\|\zeta\| e^{-b t} \\
\text { (resp. } a\|\eta\| e^{b^{\prime} t} \geq\left\|T \phi_{r} \eta\right\| \geq a^{-1}\|\eta\| e^{b t} \text { ) }
\end{gathered}
$$

where $T \phi_{t}$ is the differential of $\phi_{t}$ and $\|\cdot\|$ is the positive definite quadratic form on $\mathbf{V}_{t}^{s} \oplus \mathbf{V}_{t}^{u}$ induced by the metric $\mathscr{G}$. 
Definition 2.2. The flow $\left\{\phi_{t} \mid t \in \mathbb{R}\right\}$ is said to be hyperbolic if the following three conditions are satisfied:

(i) every one of its flow lines is hyperbolic;

(ii) the constants $a, b$ and $b^{\prime}$ can be chosen to be the same for all flow lines; and

(iii) the splitting of $T_{x} \mu$ is continuous in $x$.

The sub-bundle $\mathbf{V}^{s}$ (resp. $V^{u}$ ), over $\mathcal{M}$ with fibre $\mathbf{V}_{x}^{s}$ (resp. $\mathbf{V}_{x}^{u}$ ) is referred to as the stable (resp. unstable) sub-bundle of the flow $\left\{\phi_{t} \mid t \in \mathbb{R}\right\}$. A time-like vector field is said to be hyperbolic if its flow is hyperbolic.

Remark 2.3. The hyperbolicity of a time-like vector field is clearly preserved under a conformal change of the Lorentz metric by a function which is positive and uniformly bounded.

Remark 2.4. Our definition 2.1. can be used as well for an incomplete flow line, in which case the decomposition is over an interval on which the flow line is defined.

Remark 2.5. The subspaces $\mathbf{V}_{t}^{s}$ and $\mathbf{V}_{t}^{u}$ are determined uniquely by their defining properties, and they are flow invariant:

$$
\begin{aligned}
T \phi_{t} \cdot V_{0}^{s} & =V_{\phi_{t}}^{s} \\
T \phi_{t} \cdot V_{0}^{u} & =V_{\phi_{t}}^{u} .
\end{aligned}
$$

Remark 2.6. The dimension $k$ (resp. $l$ ) of $\mathbf{V}^{s}$ (resp. $\mathbf{V}^{u}$ ) is a constant over $\mathcal{M}$.

Remark 2.7. The hyperbolicity of the flow is preserved under a change of time by a smooth bounded positive function with bounded derivatives; i.e. if $\phi_{t}$ is a hyperbolic flow generated by a time-like vector field $\xi$ and $\alpha(x)$ is a smooth bounded positive function with bounded derivatives, then the flow $\left\{\psi_{t} \mid t \in \mathbb{R}\right\}$ generated by $\zeta(x)=\alpha(x) \xi(x)$ is also hyperbolic; the proof proceeds as in [2].

Let $\pi: T \mathscr{M} \rightarrow \mathscr{M}$ be the tangent bundle of $\mathscr{M}$ and let $\mathscr{H}$ be an arbitrary positive definite metric on $T M$. Using $\mathscr{H}$, we construct in the standard way (cf. [7]) the Whitney fine $C^{r}$-topology, $r \geq 0$, on the set $C^{r}(\mathcal{M})$ of all $C^{r}$-mappings of $M$ into itself, and a norm on the fields on each tensor bundle over $\mathcal{M}$. The Whitney fine $C^{r}$-topology so induced is independent of $\mathscr{H}$. Moreover (cf. [14]), the set of complete vector fields is open in $\Gamma^{r}(T M)$ the space of $C^{r}$-sections in $T M$ with the Whitney fine $C^{r}$-topology.

The following theorem is given as an illustration of how some of the results of Riemannian differential geometry (see e.g. [1], [3], [11]) translate to the Lorentzian case. Note, in particular, that the arguments used in the proof of this theorem do not involve any compactness conditions on $\mathcal{M}$; this generalization is necessary if one is to avoid violating the physically plausible causality conditions required in the general theory of relativity (see e.g. [4], [10], [13], [16]).

ThEOREM 2.8. Let $\left\{\phi_{l} \mid t \in \mathbb{R}\right\}$ be a hyperbolic flow defined by a time-like complete vector field $\xi$. Then the flow $\left\{\psi_{t} t \mid t \in \mathbb{R}\right\}$ defined by a time-like vector field $\zeta$ which is in a sufficiently small neighbourhood of $\xi$ in $\Gamma^{1}(T M)$ is also hyperbolic and the stable and the unstable subspaces are continuous in $\zeta$. 
Proof. We choose a positive definite metric $\mathscr{H}$ to be the metric defined by;

$$
\mathscr{H}(v, v) \equiv-\mathscr{G}(\nu, \nu)+\mathscr{G}(\bar{v}, \bar{v}),
$$

for $v=\nu+\bar{v} \in \mathbf{Z}_{x}^{1} \oplus \mathbf{V}_{x}=T_{x} \mathcal{M}$, where $\mathbf{V}_{x} \equiv \mathbf{V}_{x}^{s} \oplus \mathbf{V}_{x}^{u}$. (Note that, even if $\mathbf{V}_{x}^{s}$ and $\mathbf{V}_{x}^{u}$ are only continuous in $x$, we can always approximate them by smooth ones as close to $V_{x}^{s}$ and $V_{x}^{u}$ as we want in the $C^{1}$-topology (cf. $\S 6$ in [1])). Let $\lambda:(x, v) \in T M \rightarrow \mathbb{R}$ be a function on $T M$. We denote its derivative along the flow line $\phi_{t} x$, for $x \in \mathcal{M}$, by

$$
\left.D_{\xi} \lambda(x, v) \equiv \frac{d}{d t} \lambda\left(\phi_{t} x, T \phi_{t} v\right)\right|_{t=0}
$$

when it exists. We define now positive definite quadratic forms $\mathscr{A}, \mathscr{B}$ and $\mathscr{F}$ on $\mathrm{V}^{s}$, $\mathbf{V}^{u}$ and $\mathbf{Z}^{1}$ respectively as follows: for $x \in \mathcal{M}$,

$$
\begin{aligned}
& \mathscr{A}(x, \rho) \equiv \int_{0}^{\tau}\left\|T \phi_{t} \cdot \rho\right\|^{2} d t \quad \text { for } \rho \in \mathbf{V}_{x}^{s}, \\
& \mathscr{B}(x, \eta) \equiv \int_{0}^{\tau}\left\|T \phi_{-t} \cdot \eta\right\|^{2} d t \quad \text { for } \eta \in \mathbf{V}_{x}^{u}, \\
& \mathscr{F}(x, \nu) \equiv \frac{\|\nu\|^{2}}{\|\xi(x)\|^{2}} \quad \text { for } \nu \in \mathbf{Z}_{x}^{1},
\end{aligned}
$$

where $\|\cdot\|$ is the length of a vector induced from $\mathscr{H}$, and $\tau>0$ is a sufficiently large number which will be chosen below (see the proof, i.e. (2.18), of (2.12) and its analogue for (2.13)). We extend $\mathscr{A}, \mathscr{B}$ and $\mathscr{F}$ to non-negative definite quadratic forms on $T_{x} \mathcal{M}$ as follows: for any $v \in T_{x} \mathcal{M}$, we express $v$ uniquely as $v=\rho+\eta+\nu$ for $\rho \in \mathbf{V}_{x}^{s}, \eta \in \mathbf{V}_{x}^{u}$ and $\nu \in \mathbf{Z}_{x}^{1}$, and define $\mathscr{A}, \mathscr{B}$ and $\mathscr{F}$ on $T_{x} \mathcal{M}$ by $\mathscr{A}(x, v) \equiv \mathscr{A}(x, \rho)$, $\mathscr{B}(x, v) \equiv \mathscr{B}(x, \eta)$ and $\mathscr{F}(x, v) \equiv \mathscr{F}(x, \nu)$. Then $\mathscr{A}, \mathscr{B}$ and $\mathscr{F}$ are continuous in $x$ and we can find some positive numbers $a_{1}, a_{2}$ such that

$$
\begin{gathered}
-a_{2} \mathscr{A}(x, \rho) \leq D_{\xi} \mathscr{A}(x, \rho) \leq-a_{1} \mathscr{A}(x, \rho), \quad \text { for } \rho \in \mathbf{V}_{x}^{s} \\
a_{2} \mathscr{B}(x, \eta) \geq D_{\xi} \mathscr{B}(x, \eta) \geq a_{1} \mathscr{B}(x, \eta), \quad \text { for } \eta \in \mathbf{V}_{x}^{u} \\
D_{\xi} \mathscr{F}(x, \nu)=0, \quad \text { for } \nu \in \mathbf{Z}_{x}^{1} .
\end{gathered}
$$

Indeed, since

$$
\frac{d}{d t} \mathscr{A}\left(\phi_{t} x, T \phi_{t} \cdot \rho\right)=\frac{d}{d t} \int_{0}^{\tau}\left\|T \phi_{s+t} \cdot \rho\right\|^{2} d s=\left\|T \phi_{t+\tau} \cdot \rho\right\|^{2}-\left\|T \phi_{t} \cdot \rho\right\|^{2},
$$

for $t=0$

$$
\left.D_{\xi} \mathscr{A}(x, \rho) \equiv \frac{d}{d t} \mathscr{A}\left(\phi_{t} x, T \phi_{t} \cdot \rho\right)\right|_{t=0}=\left\|T \phi_{\tau} \cdot \rho\right\|^{2}-\|\rho\|^{2}
$$

Hence, by the hyperbolicity of $\xi$, we have

$$
-b_{2}\|\rho\|^{2} \leq D_{\xi} \mathscr{A}(x, \rho) \leq-b_{1}\|\rho\|^{2}
$$

where $b_{1} \equiv 1-a^{2} e^{-2 b \tau}$ and $b_{2} \equiv 1-a^{-2} e^{-2 b^{\prime} \tau}$. We also have

$$
b_{3}\|\rho\|^{2} \leq \mathscr{A}(x, \rho)=\int_{0}^{\tau}\left\|T \phi_{t} \cdot \rho\right\|^{2} d t \leq b_{4}\|\rho\|^{2},
$$

where $b_{3} \equiv\left(2 a^{2} b^{\prime}\right)^{-1}\left(1-e^{-2 b^{\prime} \tau}\right)$ and $b_{4} \equiv a^{2}(2 b)^{-1}\left(1-e^{-2 b \tau}\right)$. Then $b_{i}, i=1,2,3$ and 
4, are positive for some $\tau$ large enough. Thus from (2.17) and (2.18), by taking $a_{1} \equiv b_{1} / b_{4}$ and $a_{2} \equiv b_{2} / b_{3}$, we obtain (2.12). Similarly for (2.13). For (2.14),

$$
D_{\xi} \mathscr{F}(x, \nu)=\frac{d}{d t} \frac{\left\|T \phi_{t} \cdot \nu\right\|^{2}}{\left\|T \phi_{t} \cdot \xi_{(x)}\right\|^{2}}=\left.\frac{d}{d t}(\text { constant })\right|_{t=0}=0 .
$$

These forms are continuous in $x$ but are not necessarily smooth. Nevertheless (cf. e.g. [1]), one can approximate any continuous non-negative quadratic form $\lambda(x, v)$ with continuous derivative $D_{\xi} \lambda(x, v)$ by a non-negative smooth (of class $C^{\infty}$ ) quadratic form in such a way that for any $\varepsilon>0$, we can find a non-negative smooth form $\tilde{\lambda}(x, v)$ such that

$$
\begin{array}{r}
\max _{\|v\| \leq 1}|\tilde{\lambda}(x, v)-\lambda(x, v)|<\varepsilon \\
\max _{\|v\| \leq 1} \mid D_{\varepsilon} \tilde{\lambda}(x, v)-D_{\xi} \lambda(x, v) \|<\varepsilon,
\end{array}
$$

where $\|\cdot\|$ is the norm induced from $\mathscr{H}$. Indeed, since this is a local property of maps, in order to obtain such an approximation all we need is a partition of unity subordinate to a locally finite atlas of $\mathcal{M}$, (cf. $\S 6,7$ in [1]). Let us denote smooth non-negative forms which approximate our $\mathscr{A}, \mathscr{B}$ and $\mathscr{F}$ by $\mathscr{A}, \mathscr{B}$ and $\mathscr{F}$ again. Using these quadratic forms we define a positive definite metric $\mathscr{H}^{\prime}$ via the quadratic form

$$
|v|^{2} \equiv \mathscr{A}(x, v)+\mathscr{B}(x, v)+\mathscr{F}(x, v),
$$

for $v \in T_{x} \mathcal{M}, x \in \mathscr{M}$. Then, from the hypothesis, it is easy to check that $\mathscr{H}^{\prime}$ and $\mathscr{H}$ are equivalent (i.e. there is a positive constant $p$ on $M$ such that

$$
p^{-1}\|v\| \leq|v| \leq p\|v\|
$$

for all $v \in T M)$.

Since $V_{x}^{s}$ and $V_{x}^{u}$ are space-like closed subspaces in $T_{x} \mu$, the angle $\theta(x)$ with respect to the Riemann metric $\mathscr{H}$ between the subspace $\mathbf{V}_{x}^{s} \oplus \mathbf{V}_{x}^{u}$ and the null cone is positive. Let $S(x)$ be the set of space-like vectors in $T_{x} \mathcal{U}$ whose angle with the null cone is greater than or equal to $\frac{1}{2} \theta(x)$. We now consider the following closed sets; for all $x \in \mathcal{M}$,

$$
\begin{aligned}
& \mathscr{K}(x) \equiv\{v \in S(x) \mid \mathscr{A}(x, v) \geq \mathscr{B}(x, v), \mathscr{A}(x, v) \geq \mathscr{F}(x, v)\}, \\
& \mathscr{L}(x) \equiv\{v \in S(x) \mid \mathscr{B}(x, v) \geq \mathscr{A}(x, v), \mathscr{B}(x, v) \geq \mathscr{F}(x, v)\} .
\end{aligned}
$$

Then clearly $\mathbf{V}_{x}^{s} \subset \mathscr{K}(x), \mathbf{V}_{x}^{u} \subset \mathscr{L}(x)$, and for $\rho \in \mathscr{K}(x), \eta \in \mathscr{L}(x)$, we have

$$
\begin{aligned}
& \mathscr{A}(x, \rho) \leq|\rho|^{2} \leq 3 \mathscr{A}(x, \rho) \\
& \mathscr{B}(x, \eta) \leq|\eta|^{2} \leq 3 \mathscr{B}(x, \eta) .
\end{aligned}
$$

We now show that, for another time-like vector field $\zeta$ which is sufficiently close to $\xi$ in the Whitney fine $C^{1}$-topology, we still have the inequalities;

$$
\begin{aligned}
&-c_{2} \mathscr{A}(x, v) \leq D_{\zeta} \mathscr{A}(x, v) \leqq-c_{1} \mathscr{A}(x, v) \\
& c_{2} \mathscr{B}(x, v) \geq D_{\zeta} \mathscr{B}(x, v) \geq c_{1} \mathscr{B}(x, v)
\end{aligned}
$$

for some constants $c_{1}, c_{2}>0, v \in T_{x} M$, and we also have

$$
\left|D_{\zeta} \mathscr{F}(x, v)\right| \leq \frac{c}{5} \mathscr{F}(x, v) .
$$


Indeed, by understanding $D_{\xi}$ as the Lie derivation of functions on $T M$ in the direction of $\tilde{\xi}$ which is the vector field on $T \mu$ generating the flow $T \phi_{t}$, we have

$$
D_{\xi}-D_{\zeta}=D_{\xi-\zeta}
$$

Moreover, for a quadratic form $\lambda$ on $T M$, we claim that for any $\varepsilon>0$ we can find a continuous positive function $\delta: \mathscr{M} \rightarrow \mathbb{R}^{+}$on $\mathcal{M}$ such that if a vector field $\zeta$ is in a $\delta$-neighbourhood of $\xi$ in the $C^{1}$-topology (i.e. $\|(\xi(x)-\zeta(x) \|<\delta(x)$ and $\| \nabla \xi(x)-$ $\nabla \zeta(x) \|<\delta(x)$ for all $x \in \mathcal{M})$, then

$$
\left|D_{\xi-\zeta} \lambda(x, v)\right| \leq \varepsilon\|v\|^{2}, \quad \text { for all }(x, v) \in T_{x} M,
$$

where $\|\cdot\|$ is the norm induced by $\mathscr{H}$. Indeed, we write $\mathscr{M}=\bigcup_{n=1}^{\infty} K_{n}$ where, for each $n, K_{n}$ is a compact set such that $K_{n} \subseteq \operatorname{Int}\left(K_{n+1}\right)$. Set $L_{n} \equiv K_{n}-\operatorname{Int}\left(K_{n-1}\right)$ for each $n$. Then clearly $M=\bigcup_{n=1}^{\infty} L_{n}$. For each $n$, we choose a finite set of charts covering $L_{n}$, and choose one of them, say $(U, \varphi)$. Then for $(x, v) \in T U$, we can write

$$
\lambda(x, v)=\langle\lambda(x) v, v\rangle=\sum_{i, j}^{n} \lambda_{i j}(x) v^{i} v^{j}
$$

where $\langle$,$\rangle is the Euclidean inner product in the Euclidean space and \lambda(x)$ is the symmetric matrix determined by $\lambda$. If $\zeta$ is another vector field on $\mathcal{M}$, by using the Lie derivation,

$$
D_{\xi-\zeta} \lambda(x, v)=\left\langle D_{\xi-\zeta} \lambda(x) v, v\right\rangle+2\left\langle\lambda(x) v, D_{\xi-\zeta} v\right\rangle,
$$

and hence,

$$
\left|D_{\xi-\zeta} \lambda(x, v)\right| \leq\left\|D_{\xi-\zeta} \lambda(x)\right\|\|v\|^{2}+2\|\lambda(x)\|\|v\|\left\|D_{\xi-\zeta} v\right\| .
$$

Notice that by the chain rule

and

$$
D_{\xi-\zeta} \lambda_{i j}(x)=\sum_{k=1}^{n} \frac{\partial \lambda_{i j}}{\partial x_{k}}\left(\xi^{k}-\zeta^{k}\right)
$$

$$
D_{\xi-\zeta} v=D_{\xi} v-D_{\xi} v=\Gamma(x)(\xi-\zeta, v),
$$

where $\Gamma(x)$ is the matrix of Christoffel symbols and is considered here as a bilinear form on $T M$ (cf. e.g. [1], [12]). Then we can choose positive constants $C_{n}^{\prime}$ and $C_{n}^{\prime \prime}$ on $L_{n}$ such that

and

$$
\left|D_{\xi-\zeta} \lambda(x)\right| \leq C_{n}^{\prime}\|\xi-\zeta\|_{C_{L_{n}}}
$$

$$
\left|D_{\xi-\zeta} v\right| \leq C_{n}^{\prime \prime}\|\xi-\zeta\|_{C_{L_{n}}}\|v\| \text {. }
$$

And hence, from (2.32), for some constant $C_{n}$ on $L_{n}$

$$
\left|D_{\xi-\zeta} \lambda(x, v)\right| \leq C_{n}\|\xi-\zeta\|_{C_{L_{n}}^{1}}\|v\|^{2} \text {. }
$$

Define $\delta_{n}(x) \equiv \varepsilon / C_{n}>0$ for $x \in L_{n}$, and then we can construct a continuous function $\delta: M \rightarrow \mathbb{R}^{+}$such that $0<\delta(x) \leq \delta_{n}(x)$ for all $n$ and $x \in L_{n}$. From this one can derive the inequalities: $\left(2.12^{\prime}\right),\left(2.13^{\prime}\right)$ and (2.27).

We now assert that, for $t>0$,

$$
T \psi_{-t} \mathscr{K}(x) \subseteq \mathscr{K}\left(\psi_{-t} x\right)
$$

and

$$
T \psi_{t} \mathscr{L}(x) \subseteq \mathscr{L}\left(\psi_{t} x\right)
$$


where $\left\{\psi_{t} \mid t \in \mathbb{R}\right\}$ is the flow of $\zeta$. We prove for instance (2.39). If $v=\rho+\eta+\nu \in \mathscr{L}(x)$, with $v \neq 0$, where $\rho \in \mathbf{V}_{x}^{s}, \eta \in \mathbf{V}_{x}^{u}$ and $\nu \in \mathbf{Z}_{x}^{1}$, then since $\eta \neq 0$ from the condition on $\mathscr{L}(x)$, the length of space-like component $T \phi_{i}(\rho+\eta)$ increases exponentially while the length of $T \phi_{t} \nu$ remains bounded for $t>0$, and hence the angle between $v$ and the null cone is non-decreasing for $t>0$. Hence, for $t>0, T \phi_{t} v \in S(x)$. This is also true for $T \psi_{t} v$ with $\zeta$ close enough to $\xi$. Now for $t=0, \mathscr{A}(x, v) / \mathscr{B}(x, v) \leq 1$ and $\mathscr{F}(x, v) / \mathscr{B}(x, v) \leq 1$ and

$$
\begin{aligned}
D_{\zeta}\left(\frac{\mathscr{A}(x, v)}{\mathscr{B}(x, v)}\right) & =\frac{\mathscr{B}(x, v) D_{\zeta} \mathscr{A}(x, v)-\mathscr{A}(x, v) D_{\zeta} \mathscr{B}(x, v)}{\mathscr{B}(x, v)^{2}} \\
& \leq \frac{-\bar{c} \mathscr{A}(x, v) \mathscr{B}(x, v)-\bar{c} \mathscr{A}(x, v) \mathscr{B}(x, v)}{\mathscr{B}(x, v)^{2}} \\
& =\frac{-2 \bar{c} \mathscr{A}(x, v)}{\mathscr{B}(x, v)}<0 .
\end{aligned}
$$

Hence $\mathscr{A}\left(\psi_{t} x, T \psi_{t} v\right) \leq \mathscr{B}\left(\psi_{t} x, T \psi_{t} v\right)$ for $t>0$. Similarly, $\mathscr{F}\left(\psi_{t} x, T \psi_{t} v\right) \leq \mathscr{B}\left(\psi_{t} x, T \psi_{t} v\right)$ for $t>0$. This proves $(2.39) ;(2.38)$ is proved similarly.

Since, from (2.38) and (2.39), we have, for $r>t \geq 0$,

$$
\begin{aligned}
T \psi_{-r} \mathbf{V}_{\psi_{r} x}^{s} \subseteq T \psi_{-r} \mathscr{K}\left(\psi_{r} x\right) & \subseteq T \psi_{-r} \mathscr{K}\left(\psi_{t} x\right) \\
T \psi_{-r} \mathbf{V}_{\psi_{-r} x}^{u} & \subseteq T \psi_{r} \mathscr{L}\left(\psi_{-r} x\right) \subseteq T \psi_{t} \mathscr{L}\left(\psi_{-t} x\right),
\end{aligned}
$$

we can choose convergent subsequences of the sequences of linear subspaces of $T_{x} \mathcal{M} ;\left\{T \psi_{-}, \mathbf{V}_{\psi_{1} x}^{s}\right\}$ and $\left\{T \psi_{t} \mathbf{V}_{\psi_{1} x}^{u}\right\}$, as $t \rightarrow \infty$. We denote their limits by $\overline{\mathbf{v}}_{x}^{s}$ and $\overline{\mathbf{v}}_{x}^{u}$. Then, for $t>0$, we obtain from (2.41) and (2.42):

$$
\begin{aligned}
T \psi_{t} \overline{\mathbf{V}}_{x}^{s} & \subseteq \mathscr{K}\left(\psi_{t} x\right) \\
T \psi_{-t} \overline{\mathbf{v}}_{x}^{u} & \subseteq \mathscr{L}\left(\psi_{-t} x\right),
\end{aligned}
$$

which imply that $\overline{\mathbf{V}}_{x}^{s}$ and $\overline{\mathrm{V}}_{x}^{u}$ are space-like subspaces. Moreover, for $t>0$, we obtain from (2.38) and (2.39):

$$
\begin{gathered}
T \psi_{-t} \overline{\mathbf{V}}_{x}^{s} \subseteq T \psi_{-t} \mathscr{K}(x) \subseteq \mathscr{K}\left(\psi_{-t} x\right) \\
T \psi_{t} \overline{\mathbf{V}}_{x}^{u} \subseteq T \psi_{t} \mathscr{L}(x) \subseteq \mathscr{L}\left(\psi_{t} x\right)
\end{gathered}
$$

That is, for $-\infty<t<\infty$,

$$
T \psi_{t} \overline{\mathbf{V}}_{x}^{s} \subseteq \mathscr{K}\left(\psi_{t} x\right) \text { and } T \psi_{t} \overline{\mathbf{V}}_{x}^{u} \subseteq \mathscr{L}\left(\psi_{t} x\right)
$$

Therefore by $\left(2.12^{\prime}\right)$ and $\left(2.13^{\prime}\right)$, for $t \in \mathbb{R}$,

$$
\begin{array}{cc}
-c_{2} \mathscr{A}\left(\psi_{t} x, T \psi_{t} \rho\right) \leq \frac{d}{d t} \mathscr{A}\left(\psi_{t} x, T \psi_{t} \rho\right) \leq-c_{1} \mathscr{A}\left(\psi_{t} x, T \psi_{t} \rho\right) & \text { for } \rho \in \overline{\mathbf{V}}_{x}^{s}, \\
c_{2} \mathscr{B}\left(\psi_{t} x, T \psi_{t} \eta\right) \geq \frac{d}{d t} \mathscr{B}\left(\psi_{t} x, T \psi_{t} \eta\right) \geq c_{1} \mathscr{B}\left(\psi_{t} x, T \psi_{t} \eta\right) & \text { for } \eta \in \overline{\mathbf{V}}_{x}^{u}
\end{array}
$$

By integration, for $t \geq 0$,

$$
\begin{aligned}
3^{-1} e^{-c_{2} t}|\rho|^{2} & \leq\left|T \psi_{t} \rho\right|^{2} \leq 3 e^{-c_{1} t}|\rho|, \\
3 e^{c_{2} t}|\eta|^{2} & \geq\left|T \psi_{t} \eta\right|^{2} \geq 3^{-1} e^{c_{1} t}|\eta|^{2} .
\end{aligned}
$$


Since $\mathscr{H}^{\prime}$ and $\mathscr{H}$ are equivalent, we obtain, for $t \geq 0$,

$$
\begin{aligned}
p^{-1} e^{-c_{2} t}\|\rho\|^{2} & \leq\left\|T \psi_{t} \rho\right\|^{2} \leq p e^{-c_{2} t}\|\rho\|^{2}, \\
p e^{c_{2} t}\|\eta\|^{2} & \geq\left\|T \psi_{t} \eta\right\|^{2} \geq p^{-1} e^{c_{1} t}\|\eta\|^{2},
\end{aligned}
$$

where $p$ is some positive constant. In this way, we obtained the contracting and expanding subspaces $\overline{\mathbf{V}}_{x}^{s}$ and $\overline{\mathbf{V}}_{x}^{u}$, respectively, for the flow $\psi_{t}$. The inequalities (2.52) and (2.53) also show that

$$
\overline{\mathbf{V}}_{x}^{s} \cap \overline{\mathbf{V}}_{x}^{u}=\{0\}
$$

and moreover we have

$$
\overline{\mathbf{Z}}_{x}^{1} \cap\left(\overline{\mathbf{V}}_{x}^{s} \oplus \overline{\mathbf{V}}_{x}^{u}\right)=\{0\},
$$

where $\overline{\mathbf{Z}}_{x}^{1}$ is the 1-dimensional time-like subspace generated by $\zeta(x)$. By counting the dimensions of these subspaces, we have

$$
T_{x} \mathcal{M}=\overline{\mathbf{Z}}_{x}^{1} \oplus \overline{\mathbf{V}}_{x}^{s} \oplus \overline{\mathbf{V}}_{x}^{u}
$$

\section{Definition of the time-like geodesic flow}

In this and the next sections $(M, g)$ is supposed to be a space-time which is time-like geodesically complete, unless otherwise stated. We denote by $\nabla$ the Levi-Civita connection associated to $g$, and by $\mathbf{D}$ the connection map (cf. [12]) from $T(T M)$ to $T \mathbf{M}$ induced by $\nabla$; we have then the commutative diagram:

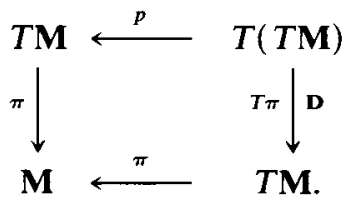

The connection map $\mathbf{D}$ determines a splitting of the bundle $p: T(T \mathbf{M}) \rightarrow T M$ into two sub-bundles over $T \mathbf{M}$, namely $\operatorname{Hor} T(T \mathbf{M})=\operatorname{Ker} \mathbf{D}$ and $\operatorname{Ver} T(T \mathbf{M})=\operatorname{Ker} T \pi$. Over each $X \in T M$ we thus have the fibre decomposition

$$
T_{X}(T \mathbf{M})=\operatorname{Hor} T_{X}(T \mathbf{M}) \oplus \operatorname{Ver} T_{X}(T M)
$$

where Hor $T_{X}(T \mathrm{M})$ [resp. Ver $T_{X}(T \mathbf{M})$ ] is isomorphic to $T_{\pi(X)} \mathbf{M}$; the isomorphism being given by the restriction of $T \pi$ [resp. D] to Hor $T_{X}(T M)$ [resp. Ver $T_{X}(T M)$ ]. (Henceforth, we implicitly identify, for $\xi=\xi^{h} \oplus \xi^{v} \equiv\left(\xi^{h}, \xi^{v}\right), \xi^{h} \in \operatorname{Hor} T_{X}(T \mathbf{M})$ [resp. $\xi^{v} \in \operatorname{Ver} T_{X}(T \mathbf{M})$ ] with $T_{X} \pi \xi^{h}$ [resp. $\left.\left.\mathbf{D} \xi\right] \in T_{\pi(X)} \mathbf{M}\right)$. This splitting allows one to define a pseudo-Riemannian metric $g^{T}$ and a symplectic structure $\omega^{T}$ on $T \mathbf{M}$ :

$$
\begin{gathered}
g_{X}^{T}\left(\xi_{1}, \xi_{2}\right) \equiv g_{x}\left(\xi_{1}^{h}, \xi_{2}^{h}\right)+g_{x}\left(\xi_{1}^{v}, \xi_{2}^{v}\right), \\
\omega_{X}^{T}\left(\xi_{1}, \xi_{2}\right) \equiv g_{x}\left(\xi_{1}^{h}, \xi_{2}^{v}\right)-g_{x}\left(\xi_{1}^{v}, \xi_{2}^{h}\right),
\end{gathered}
$$

where $X \in T M, \quad x=\pi(X), \quad$ and $\quad($ for $i=1,2) \quad \xi_{i}=\left(\xi_{i}^{h}, \xi_{i}^{v}\right) \in \operatorname{Hor} T_{X}(T \mathbf{M}) \oplus$ Ver $T_{X}(T M)$.

Note that $\omega^{T}$ is the pull-back of the canonical symplectic form $\omega$ on $T^{*} \mathbf{M}$, via the metric $g$. Note further that $\omega_{X}^{T}$ equips the $2 n$-dimensional vector space $T_{X}(T M)$ with a symplectic structure, and that $\omega_{X}^{T}$ vanishes identically on each of the $n$ dimensional subspaces Hor $T_{X}(T M)$ and $\operatorname{Ver} T_{X}(T M)$; these are thus complemen- 
tary Lagrangian subspaces of $T_{X}(T M)$. Note finally that $\pi$ is a surjective submersion and that for each $X \in T \mathbf{M}, T_{X} \pi:$ Hor $T_{X}(T \mathbf{M}) \rightarrow T_{\pi(X)} \mathbf{M}$ is an isometry, i.e. $\pi$ is an isometric submersion. Upon using the splitting (3.2), we can rewrite the Hamiltonian vector field $\xi_{H}$ on $T \mathbf{M}$ for the Hamiltonian function $H: X \in T M \mapsto \frac{1}{2} g(X, X) \in$ $\mathbb{R}$ in the form

$$
\xi_{H}: X \in T \mathbf{M} \mapsto(X, 0) \in \text { Hor } T_{X}(T \mathbf{M}) \oplus \operatorname{Ver} T_{X}(T \mathbf{M})
$$

When $(M, g)$ is complete in every direction, the flow $\left\{\phi_{t} \mid t \in \mathbb{R}\right)$ of $\xi_{H}$ satisfies the following properties for all $X \in T M$ : (i) $\phi_{t} X$ is well-defined for all $t \in \mathbb{R}$; (ii) $\left\{\gamma_{X}(t) \equiv \pi \phi_{t} X \mid t \in \mathbb{R}\right\}$ is the geodesic on $\mathbf{M}$ determined by $\gamma_{X}(0)=\phi(X)$ and $\dot{\gamma}_{X}(0)=$ $X$; (iii) $\phi_{t}$ is symplectic for all $t \in \mathbb{R}$; (iv) $\left\{\phi_{t} X \mid t \in \mathbb{R}\right\}$ is a gcodesic in $T M$; (v) for $a<0, H^{-1}(a)$ is a submanifold of codimension 1 and $\left\{\phi_{t} X \mid t \in \mathbb{R}\right\} \subseteq H^{-1}(a)$ whenever $H(X)=a$; (vi) for $a, b \in \mathbb{R}$ with $a \cdot b>0, H^{-1}(a)$ and $H^{-1}(b)$ are isomorphic. Consequently, the study of time-like geodesic flows can be carried out, without loss of generality, on one of the connected component $\mathfrak{\Im} M$ of $H^{-1}\left(-\frac{1}{2}\right)$; we choose $\mathcal{M} \equiv \mathfrak{F} M$ to be sub-bundle of $T M$, the elements of which are the future-directed, time-like, unit tangent vectors to $M$.

Remark 3.1. Properties (ii)-(vi) do not require the geodesic completeness of (M; $g$ ). Neither does the following lemma; however, if a geodesic line is incomplete, the argument should be understood as extending only up to points where the geodesic line (or flow line) is defined.

LEMMA 3.2. The restriction $\mathscr{G}$ of $\mathrm{g}^{T}$ to $\mathfrak{\Im} \mathbf{M}$ is a Lorentz metric; and there exists a T申-invariant splitting

$$
T(\mathfrak{I} \mathbf{M})=T^{\mathbf{l}}(\mathfrak{I} \mathbf{M}) \oplus T^{c}(\mathfrak{I} \mathbf{M})
$$

such that: $T^{\mathbf{1}}(\mathfrak{I} \mathbf{M})$ is a line bundle over $\mathfrak{J} \mathbf{M}$, on which $\mathscr{G}$ is negative-definite; $T^{c}(\mathfrak{I} \mathbf{M})$ is a sub-bundle over $\mathfrak{I} \mathbf{M}, \mathscr{G}$-orthogonal to $T^{\mathbf{l}}(\mathfrak{I} \mathbf{M})$; and $\omega^{T}$ is non-degenerate over $T^{c}(\mathfrak{S} \mathbf{M})$.

Proof. For $\mathrm{X} \in \mathfrak{\Im} \mathbf{M}$ we define $T_{X}^{k}(\mathfrak{I} \mathbf{M})$, with $k=1,2, c$, as follows: $T_{X}^{1}(\mathfrak{I} \mathbf{M})$ is the one-dimensional subspace of $T_{X}(\mathfrak{\Im} \mathbf{M})$ generated by $\xi_{H}(X)=(X, 0) ; T_{X}^{2}(\mathfrak{\Im} \mathbf{M})$ is the two-dimensional subspace of $T_{X}(T M)$ spanned by $(X, 0) \in \operatorname{Hor} T_{X}(T M)$ and $(0, X) \in \operatorname{Ver} T_{X}(T \mathbf{M}) ; T_{X}^{c}(\mathfrak{I} M)$ is the $\omega^{T}$-orthogonal complement in $T_{X}(T M)$ of $T_{X}^{2}(\mathfrak{I} \mathbf{M})$. Note that $T_{X}^{c}(\mathfrak{I} \mathbf{M}) \subseteq T_{X}(\mathfrak{I} \mathbf{M})$, and since, for $X \in \mathfrak{I} \mathbf{M}$,

$$
\left.g_{X}^{T}((X, 0)),(X, 0)\right)=g_{\pi(X)}(X, X)=-1,
$$

$g_{X}^{T}$ is a negative-definite on $T^{1}(\mathfrak{\Im} \mathbf{M})$. For every $\xi=\left(\xi^{h}, \xi^{v}\right) \in T_{X}^{c}(\mathfrak{I} \mathbf{M}]$, we have

$$
\begin{aligned}
& 0=\omega_{X}^{T}\left((X, 0),\left(\xi^{h}, \xi^{v}\right)\right)=g_{\pi(X)}\left(X, \xi^{v}\right) \\
& 0=\omega_{X}^{T}\left((0, X),\left(\xi^{h}, \xi^{v}\right)\right)=-g_{\pi(X)}\left(X, \xi^{h}\right)
\end{aligned}
$$

so that both $\xi^{h}$ and $\xi^{v}$ are space-like; hence $g_{X}^{T}$ is positive-definite on $T_{X}^{c}(\mathfrak{I} \mathbf{M})$. Since $\omega_{X}^{T}$ is non-degenerate on $T_{X}^{2}(\mathfrak{J} \mathbf{M})$, so is it on $T_{X}^{c}(\mathfrak{J M})$, and therefore we have

$$
T_{X}(\mathfrak{I} \mathbf{M})=T_{X}^{1}(\mathfrak{I} \mathbf{M}) \oplus T_{X}^{c}(\mathfrak{I} \mathbf{M})
$$

Hence the restriction $\mathscr{G}$ of $g^{T}$ to $\mathfrak{\Im} \mathbf{M}$ is a Lorentz metric. Let $T^{k}(\mathfrak{I} \mathbf{M})(k=1, c)$ be the sub-bundle of $T(\mathfrak{F} \mathbf{M})$ over $\mathfrak{\Im} M$ with fibres $T_{X}^{k}(\mathfrak{I} \mathbf{M})$. All the claims of the lemma follow then straightforwardly, except perhaps for the fact that the splitting (3.6) is 
$T \phi$-invariant. To show the latter recall, for setting the notation, that a Jacobi field $\{J(t) \mid t \in \mathbb{R}\}$ over a geodesic $\{\gamma(t) \mid t \in \mathbb{R}\}$ is a vector field satisfying the Jacobi equation

$$
\nabla_{\dot{\gamma}}^{2} J(t)+R(J(t), \dot{\gamma}(t)) \dot{\gamma}(t)=0,
$$

where $R$ is the Riemann curvature tensor of the metric $g$. In particular, for $X \in \mathfrak{\Im} M$, the map

$$
J(t) \in T_{\pi \phi_{t} X} \mathbf{M} \mapsto \eta(t) \in T_{\phi_{t} X}(T \mathbf{M})
$$

with $\eta(t) \equiv\left(J(t), \nabla_{\dot{\gamma}} J(t)\right) \in$ Hor $T_{\phi_{1} X}(T \mathbf{M}) \oplus \operatorname{Ver} T_{\phi_{l} X}(T \mathbf{M})$ defines a bijection between the Jacobi fields over the geodesic $\left\{\gamma_{X}(t)=\pi \phi_{t} X \mid t \in \mathbb{R}\right\}$ and the $T \phi$ invariant vector fields over the flow line $\left\{X(t)=\phi_{t} X \mid t \in \mathbb{R}\right\}$ (see [12], and notice that the proof given there in fact does not depend on the condition that the metric be Riemannian). Consider then the Jacobi fields tangential to $\left\{\gamma_{X}(t) \mid t \in \mathbb{R}\right\}$ given by $\left\{\dot{\gamma}_{X}(t)=\phi_{t} X \mid t \in \mathbb{R}\right\}$ and $\left\{t \dot{\gamma}_{X}(t)=t \phi_{t} X \mid t \in \mathbb{R}\right\}$; they correspond, via (3.11), to the $T \phi$-invariant vector fields over $\{X(t) \mid t \in \mathbb{R}\}$ given, for all $t \in \mathbb{R}$, by:

$$
\begin{aligned}
\left(\dot{\gamma}_{X}(t), 0\right) & =T \phi_{t}(X, 0) \\
\left(t \dot{\gamma}_{X}(t), \dot{\gamma}_{X}(t)\right) & =T \phi_{t}(0, X) .
\end{aligned}
$$

This implies that $T^{1}(\mathfrak{S} \mathbf{M}), T^{2}(\mathfrak{I} \mathbf{M})$ and thus $T^{c}(\mathfrak{I} \mathbf{M})$ are stable under $T \phi_{t}$ for all $t \in \mathbb{R}$, i.e. that the splitting (3.6) is $T \phi$-invariant.

Remark 3.3. The Jacobi fields orthogonal to $\left\{\gamma_{X}(t) \mid t \in \mathbb{R}\right\}$ correspond to the $T \phi$ invariant vector fields in $T^{c}(\mathfrak{\Im M})$ given by (3.11) with $J(t) \in \dot{\gamma}_{X}(t)^{\perp}$.

Remark 3.4. The splitting (3.6) can be refined to a $G$-orthogonal decomposition of $T(\mathfrak{J} \mathbf{M})$ into sub-bundles over $\mathfrak{F} \mathbf{M}$, namely

$$
T(\mathfrak{I} \mathbf{M})=T^{1}(\mathfrak{I} \mathbf{M}) \oplus \operatorname{Hor} T^{c}(\mathfrak{I} \mathbf{M}) \oplus \operatorname{Ver} T^{c}(\mathfrak{I} \mathbf{M})
$$

where the fibres

$$
\begin{aligned}
& \text { Hor } T_{X}^{c}(\mathfrak{I} \mathbf{M})=T_{X}^{c}(\mathfrak{I} \mathbf{M}) \cap \text { Hor } T_{X}(T \mathbf{M}) \\
& \operatorname{Ver} T_{X}^{c}(\mathfrak{J} \mathbf{M})=T_{X}^{c}(\mathfrak{\Im} \mathbf{M}) \cap \operatorname{Ver} T_{X}(T \mathbf{M})
\end{aligned}
$$

for Lagrangean subspaces $T_{X}^{c}(\mathfrak{⿰} \mathbf{M})$.

Definition 3.5. The flow $\left\{\phi_{t} \mid t \in \mathbb{R}\right\}$ of $\xi_{H}$, when restricted to $(\mathfrak{F} \mathbf{M}, \mathscr{G})$, is called the time-like geodesic flow of the space-time $(\mathbf{M}, g)$.

The properties of the flows just defined are studied in the next section; in particular, their hyperbolicity (and its stability) is proven when the time-like sectional curvature of the Lorentz metric is positive and satisfies certain boundedness conditions that we specify.

\section{Hyperbolicity for time-like geodesic flows}

THEOREM 4.1. Let $\left\{\phi_{t} \mid t \in \mathbb{R}\right\}$ be the time-like geodesic flow of a space-time $(\mathbf{M}, g)$. Then the flow lines $\left\{\phi_{t} X_{0} \mid t \in \mathbb{R}\right\}$ are hyperbolic for each $X_{0} \in \mathfrak{F} \mathbf{M}$ that satisfies the following condition: there exist constants $K_{1}$ and $K_{2}$ (possibly depending on $X_{0}$ ) such that for every 2-plane $\sigma$, tangent to the geodesic line $\left\{\gamma(t)=\pi \phi_{t} X_{0} \mid t \in \mathbb{R}\right\}$,

$$
0<K_{1}^{2} \leq K(\sigma) \leq K_{2}^{2},
$$

where $K(\sigma)$ is the sectional curvature of $\sigma$. 
Proof. Let $X_{0}$ be a vector in $\mathfrak{\Im} M$ satisfying condition $(4.1) ; X(\tau) \equiv \phi_{\tau} X_{0} ; \omega_{\tau}^{c}$ the symplectic form obtained by restricting $\omega^{T}$ to $T_{X(\tau)}^{c}(\mathfrak{J} \mathbf{M})$; and

$$
T_{X(\tau)}^{c}(\mathfrak{I M})=\text { Hor } T_{X(\tau)}^{c}(\mathfrak{I} \mathbf{M}) \oplus \operatorname{Ver} T_{X(\tau)}^{c}(\mathfrak{I} \mathbf{M})
$$

be the decomposition of $T_{X(\tau)}^{c}(\mathfrak{J} \mathbf{M})$ into Lagrangean subspaces obtained in the proof of lemma 3.2 (see also (3.14)-(3.16)). Since both of these subspaces of $T_{X(r)}^{c}(\mathfrak{\Im} \mathbf{M})$ with

$$
\omega_{\tau}^{c}(\eta, \zeta)=\left\langle\eta^{h}, \zeta^{v}\right\rangle-\left\langle\eta^{v}, \zeta^{h}\right\rangle
$$

for all $\eta, \zeta \in T_{X(\tau)}^{c}(\mathfrak{J M})$ are isomorphic to a Euclidean space $\left(\mathbb{R}^{n-1},\langle\rangle,\right)$, the map

$$
A \mapsto L_{A} \equiv\left\{(\eta, A \eta) \in T_{X(\tau)}^{c}(\mathfrak{\Im} \mathbf{M}) \mid \eta \in \text { Hor } T_{X(\tau)}^{c}(\mathfrak{\Im} \mathbf{M})\right\}
$$

establishes a bijection between the symmetric operators $A$ acting on $\left(\mathbb{R}^{n-1},\langle\rangle,\right)$ and the Lagrangean subspaces $L$ of $\left(T_{X(\tau)}^{c}(\mathfrak{\Im} \mathbf{M}), \omega_{\tau}^{c}\right)$ such that $P L=$ Hor $T_{X(\tau)}^{c}(\mathfrak{\Im} \mathbf{M})$; where $P$ is the projection of $T_{X(\tau)}^{c}(\tilde{\mathfrak{J}} \mathbf{M})$ onto Hor $T_{X(\tau)}^{c}(\mathfrak{I} \mathbf{M})$ corresponding to the decomposition (4.2). Under the inverse of the map (4.4), the image $A_{L}$ of a Lagrangean subspace $L=\left\{\left(\eta^{h}, \eta^{v}\right)\right\}$ is given by

$$
A_{L} \eta^{h}=\eta^{v}
$$

If $K_{1}=K_{2}=K$, the Jacobi equation (3.10) reduces to $\nabla^{2} J(t)-K^{2} J(t)=0$ (where we will henceforth denote simply $\nabla$ the covariant derivative $\nabla_{\dot{\gamma}(t)} \equiv \nabla_{X(t)}$ along the geodesic $\left.\left\{\gamma(t)=\pi \phi_{t} X_{0} \mid t \in \mathbb{R}\right\}\right)$; hence one obtains the stable and unstable fields explicitly as

$$
\begin{aligned}
& \zeta_{s}(t)=e^{-t K} \zeta_{s}(0) \\
& \zeta_{u}(t)=e^{t K} \zeta_{u}(0)
\end{aligned}
$$

Now we assume $K_{1}<K_{2}$ and choose a real $\tau$ and a symmetric operator $A_{\tau}$ such that the spectrum of $A_{\tau}$, denoted by $\operatorname{Sp}\left(A_{\tau}\right)$, satisfies

$$
\operatorname{Sp}\left(A_{\tau}\right) \subseteq\left[K_{1}, K_{2}\right]
$$

and let $L_{\tau}$ be the Lagrangean subspace corresponding to $A_{\tau}$ via (4.4). Since $T \phi_{t-\tau}$ is symplectic for every $t \in \mathbb{R}$ and $T^{c}(\mathfrak{I} \mathbf{M})$ is $T \phi$-stable, $L_{\tau}(t) \equiv T \phi_{t-\tau} L_{\tau}$ is a Lagrangean subspace of $T_{X(t)}^{c}(\Im M)$. Since $T \phi$ is continuous in $t$, we know that there exists $\varepsilon>0$ such that for $t \in(-\varepsilon, \varepsilon)$ the image of $L_{\tau}(t)$ through $P$ is Hor $T_{X(t)}^{c}(\mathfrak{F} M)$ (we shall see later in the proof that the argument can be extended to all $t \in \mathbb{R}) . L_{\tau}(t)$ thus defines, through (4.5), a symmetric operator $A_{\tau}(t)$ acting on Hor $T_{X(t)}^{c}(\mathfrak{\Im} \mathbf{M})$ such that

$$
\left(\eta_{t}^{h}, A_{\tau}(t) \eta_{t}^{h}\right) \in L_{\tau}(t) \quad \text { for } \eta_{t}^{h} \in \operatorname{Hor} T_{X(t)}^{c}(\mathfrak{I M}) .
$$

For every $\eta^{h} \in$ Hor $T_{X(\tau)}^{c}(\mathfrak{I} M), \eta(t) \equiv T \phi_{t-\tau}\left(\eta^{h}, A_{\tau} \eta^{h}\right)$ belongs to $L_{\tau}(t)$, and can therefore be written in the form $\eta(t)=\left(\eta_{t}^{h}, A_{\tau}(t) \eta_{t}^{h}\right)$ with $\eta_{t}^{h} \equiv \eta(t)^{h} \in$ Hor $T_{X(t)}^{c}(\widetilde{\Im} \mathbf{M})$. This defines a $T \phi$-invariant vector field $\{\eta(t) \mid t \in(-\varepsilon, \varepsilon)\}$ over the flow line $\left\{X(t)=\phi_{t} X_{0} \mid t \in(-\varepsilon, \varepsilon)\right\}$. Furthermore $\left\{J(t) \equiv \eta_{t}^{h} \mid t \in(-\varepsilon, \varepsilon)\right\}$ is a Jacobi field over the geodesic $\left\{\gamma(t)=\pi \phi_{t} X_{0} \mid t \in(-\varepsilon, \varepsilon)\right\}$ and satisfies

$$
\nabla J(t)=A_{\tau}(t) J(t)
$$

From the Jacobi equation for $J$, one obtains the Riccati-type differential equation

$$
\nabla A_{\tau}(t)+A_{\tau}(t)^{2}+R_{X(\tau)}=0
$$


where $R_{X(\tau)} J(t)=R(J(t), X(t)) X(t)$. Since $g(X(t), X(t))=-1$ and $g(J(t)$, $X(t))=0$, we have

$$
g\left(R_{X(\tau)} J(t), J(t)\right)=-K(\sigma\{J(t), X(t)\}) g(J(t), J(t))
$$

so that (4.1) implies

$$
\operatorname{Sp}\left(R_{X(\tau)}\right) \subseteq\left[-K_{2}^{2},-K_{1}^{2}\right]
$$

Consequently, for $(J(t), \nabla J(t))=\left(J(t), A_{\tau}(t) J(t)\right) \in L_{\tau}(t)$, we have:

$$
\frac{1}{2} \frac{d^{2}}{d t^{2}}\|J(t)\|^{2} \geq K_{1}^{2}\|J(t)\|^{2}+\left\|A_{\tau}(t) J(t)\right\|^{2} .
$$

Since $J(t)$ is smooth on $\phi_{t} X_{0}, A_{\tau}(t)$ does not become infinite in a finite time, and is therefore defined for all $t \in \mathbb{R}$. Note also that $\left\langle A_{\tau}(t) J_{1}(t), J_{2}(t)\right\rangle=\left\langle J_{1}(t), A_{\tau}(t) J_{2}(t)\right\rangle$, for all $t \in \mathbb{R}$. We now show

$$
\operatorname{Sp}\left(A_{\tau}(t)\right) \subseteq\left[K_{1}, K_{2}\right] \quad \text { for } t \geq \tau .
$$

Let $\alpha(t)$ be the largest eigenvalue of $A_{\tau}(t), \varepsilon>0$ be arbitrarily small, and $t_{\varepsilon}$ be the first value of $t>\tau$ such that $\alpha\left(t_{\varepsilon}\right)=K_{2}+\varepsilon$. From (4.11) we obtain

$$
\dot{\alpha}\left(t_{\varepsilon}\right) \leq-\alpha\left(t_{\varepsilon}\right)^{2}+K_{2}^{2}\left(t_{\varepsilon}\right),
$$

where $K_{2}^{2}\left(t_{\varepsilon}\right) \leq K_{2}^{2}$ (see (4.13)) is the largest eigenvalue of $-R_{X\left(t_{\varepsilon}\right)}$. From this remark it follows that $\alpha(t) \leq K_{2}$ for all $t>\tau$. One can show similarly that $\beta(t)$, the smallest eigenvalue of $A_{\tau}(t)$, is uniformly bounded below by $K_{1}$.

We next show that for all $\sigma \leq \tau$, the symmetric operators $A_{\sigma}(t)$ and $A_{\tau}(t)$ constructed as above satisfy

$$
\left\|A_{\tau}(t)-A_{\sigma}(t)\right\| \leq\left\|A_{\tau}-A_{\sigma}(\tau)\right\| e^{-2 K_{1}(t-\tau)},
$$

where $\|\cdot\|$ is the operator-norm induced by the restriction of $\mathscr{G}$ to $T^{\mathrm{c}}(\mathfrak{I} \mathbf{M})$. Indeed, with $B_{\tau \sigma}(t) \equiv A_{\tau}(t)-A_{\sigma}(t)$, we obtain from (4.11) that

$$
\nabla B_{\tau \sigma}(t)=-A_{\tau}(t) B_{\tau \sigma}(t)-B_{\tau \sigma}(t) A_{\sigma}(t) .
$$

We can, therefore, write $B_{\tau \sigma}(t)$ in the form

$$
B_{\tau \sigma}(t)=C_{\tau \sigma}(t)\left[A_{\tau}-A_{\sigma}(\tau)\right] D_{\tau \sigma}(t)
$$

where $C_{\tau \sigma}$ and $D_{\tau \sigma}$ satisfy the differential equations

$$
\begin{aligned}
& \nabla C_{\tau \sigma}(t)=-A_{\tau}(t) C_{\tau \sigma}(t) \\
& \nabla D_{\tau \sigma}(t)=-D_{\tau \sigma}(t) A_{\sigma}(t),
\end{aligned}
$$

with initial conditions $C_{\tau \sigma}(\tau)=I=D_{\tau \sigma}(\tau)$. Consequently,

$$
\begin{aligned}
& \frac{d}{d t}\left\|C_{\tau \sigma}(t)\right\|^{2} \leq-2 K_{1}\left\|C_{\tau \sigma}(t)\right\|^{2} \\
& \frac{d}{d t}\left\|D_{\tau \sigma}(t)\right\|^{2} \leq-2 K_{1}\left\|D_{\tau \sigma}(t)\right\|^{2}
\end{aligned}
$$

with $\left\|C_{\tau \sigma}(\tau)\right\|=1=\left\|D_{\tau \sigma}(\tau)\right\|$. We have thus, for $t \geq \tau$ :

$$
\begin{aligned}
\left\|C_{\tau \sigma}(t)\right\| & \leq e^{-K_{1}(t-\tau)} \\
\left\|D_{\tau \sigma}(t)\right\| & \leq e^{-K_{1}(t-\tau)} .
\end{aligned}
$$

Hence (4.17) follows from (4.19) and (4.24), (4.25). 
It follows that, from (4.15) and (4.17),

$$
A^{u}(t) \equiv \lim _{\tau \rightarrow-\infty} A_{\tau}(t)
$$

exists, and defines a symmetric operator on Hor $T_{X(t)}^{c}(\mathfrak{I} \mathbf{M})$ with

$$
\operatorname{Sp}\left\{A^{u}(t)\right\} \subseteq\left[K_{1}, K_{2}\right] \text {. }
$$

We define, for all $t \in \mathbb{R}$, the Lagrangean subspace $V_{t}^{u}$ of $T_{X(t)}^{c}(\mathfrak{F} M)$ by

$$
\mathbf{V}_{t}^{u} \equiv\left\{\left(\eta^{h}(t), A^{u}(t) \eta^{h}(t)\right) \mid \eta^{h}(t) \in \text { Hor } T_{X(t)}^{c}(\mathfrak{F} \mathbf{M})\right\}
$$

and then in order to obtain the stable subspace $\mathbf{V}_{t}^{s}$ of $T_{X(t)}^{c}(\mathfrak{S} \mathbf{M})$ we consider the flow line $\phi_{t}^{\prime} X_{0} \equiv \phi_{-t} X_{0}$ which is generated by $-\zeta$ and define, for $t \in \mathbb{R}$,

$$
\mathbf{V}_{t}^{s} \equiv\left(\mathbf{V}^{\prime}\right)_{-t}^{u}
$$

where $\left(V^{\prime}\right)_{r}^{u}$, for $r \in \mathbb{R}$, is the unstable subpace in $T_{X(-r)}^{c}(\mathfrak{I} \mathbf{M})$ obtained from the flows $\phi_{r}^{\prime}$ and $T \phi_{r}^{\prime}$.

We now show that $V_{t}^{u}$ is $T \phi$-invariant and satisfies (2.4). Indeed, for large negative number $\tau$, choose an element

$$
\eta_{\tau}(t) \equiv\left(J(t), A_{\tau}(t) J(t)\right) \in L_{\tau}(t)
$$

where $J(t)$ is a Jacobi field which yields $\eta(t) \in V_{t}^{u}$ as $\tau \rightarrow-\infty$. Then

and

$$
T \phi_{t_{0}} \eta_{\tau}(t)=\eta_{\tau}\left(t+t_{0}\right)=\left(J\left(t+t_{0}\right), A_{\tau}\left(t+t_{0}\right) J\left(t+t_{0}\right)\right)
$$

$$
T \phi_{t_{0}} \eta(t)=T \phi_{t_{0}}\left(\lim _{\tau \rightarrow-\infty} \eta_{\tau}(t)\right)=\lim _{\tau \rightarrow-\infty} T \phi_{t_{0}} \eta_{\tau}(t)=\eta\left(t+t_{0}\right) \in \mathbf{V}_{t+t_{0}}^{u} .
$$

From the following inequalities which are immediate consequences of our previous estimates

$$
\begin{gathered}
2 K_{1}\|J(t)\|^{2} \leq 2\left\langle J(t), A^{u}(t) J(t)\right\rangle=\frac{d}{d t}\|J(t)\|^{2} \leq 2 K_{2}\|J(t)\|^{2} \\
K_{1}^{2}\|J(t)\|^{2} \leq\left\|A^{u}(t) J(t)\right\|^{2}=\left\|\left.\nabla J(t)\right|^{2} \leq K_{2}^{2}\right\| J(t) \|^{2},
\end{gathered}
$$

we get (for $t>0$ ):

and

$$
e^{K_{1} t}\left\|J\left(t_{0}\right)\right\| \leq\left\|J\left(t+t_{0}\right)\right\| \leq e^{K_{2} t}\left\|J\left(t_{0}\right)\right\|
$$

$$
\frac{K_{1}}{K_{2}} e^{K_{1} t}\left\|\nabla J\left(t_{0}\right)\right\| \leq\left\|\nabla J\left(t+t_{0}\right)\right\| \leq \frac{K_{2}}{K_{1}} e^{K_{2} t}\left\|\nabla J\left(t_{0}\right)\right\| .
$$

Hence, upon noticing that $K_{1} \leq K_{2},(4.35)$ and (4.36) give:

$$
\frac{K_{1}}{K_{2}} e^{K_{1} t}\left\|\eta\left(t_{0}\right)\right\| \leq\left\|T \phi_{1} \eta\left(t_{0}\right)\right\| \leq \frac{K_{2}}{K_{1}} e^{K_{2} t}\left\|\eta\left(t_{0}\right)\right\|,
$$

which is the inequality (2.4).

Therefore, $\mathbf{V}_{t}^{u}\left(\right.$ resp. $\left.\mathbf{V}_{t}^{s}\right)$ is the unstable (resp. stable) subspace of $T_{X(\tau)}^{c}(\mathfrak{I} \mathbf{M})$ over the hyperbolic flow line $\left\{\phi_{1} X_{0} \mid t \in \mathbb{R}\right\}$.

COROLLARY 4.2. The hyperbolicity of the flow line still holds for incomplete flow lines under the same curvature condition as in theorem 4.1.

Indeed, in the proof of theorem 4.1 we choose $\tau$ to be an interior point of the domain of the geodesic line $\gamma_{X}$ and in (4.26) define $A^{u}(t) \equiv \lim _{\tau \rightarrow a^{+}} A_{\tau}(t)$, where 
$\gamma_{X}:(a, b) \rightarrow \mathbf{M}$ is a maximal geodesic with initial conditions $\gamma_{X}(0)=\pi(X)$ and $\dot{\gamma}_{X}(0)=X$.

Therefore, if the timelike sectional curvature is positive and uniformly bounded above, and below away from zero, on a compact subset of $(\mathbf{M}, g)$ we still have the hyperbolicity of the geodesic flow at least on this compact set.

Corollary 4.3. Let $(\mathbf{M}, g)$ be a space-time. Suppose that every flow line of the time-like geodesic flow satisfies the conditions in theorem 4.1. Then the time-like geodesic flow of $(\mathbf{M}, g)$ is hyperbolic in the weakened sense that the constants $a, b$ and $b^{\prime}$ in the definition of hyperbolicity may depend continuously on $X \in \mathfrak{F} \mathbf{M}$.

Proof. Since the symmetric operator $A^{u}(\tau)$ on Hor $T_{X_{0}(\tau)}^{c}(\mathfrak{I} M)$ was obtained as the uniform limit of $A_{\tau}(t)$ as $\tau \rightarrow-\infty$, it will depend continuously on $X$ if we choose $A_{\tau}$ smooth in $X \in \mathfrak{F} \mathbf{M}$ where $X$ is in a small neighbourhood of $X_{0}$. Note that the spectrum of $A_{\tau}$ changes smoothly with $X$ in a small neighbourhood of $X$ since the curvature of time-like 2-planes varies smoothly.

Remark 4.4. In particular, when the time-like sectional curvature of $(\mathbf{M}, g)$ is positive and uniformly bounded on $\mathbf{M}$, the proofs of theorem 4.1 and corollary 4.3 show that the flow is hyperbolic in the usual sense.

Remark 4.5. It follows from theorem 2.8 that a time-like flow obtained by a sufficiently small perturbation of the time-like geodesic flow on a space-time of positive and uniformly bounded curvature is also hyperbolic.

Remark 4.6. When $\operatorname{dim} \mathbf{M} \geq 3$, the supplementary assumption made in remark 4.4 is very strong; indeed, the sectional curvature of $\mathbf{M}$ must then be constant (cf. [9]).

Sсноцium 4.7. There exists a space-time $(\mathbf{M}, g)$ that satisfies the assumptions of theorem 4.1 for all $x_{0} \in \mathfrak{I} M$ but that still have unbounded positive time-like sectional curvature.

Proof. One example will suffice. Let $\mathbf{M}=\left\{\left(x_{1}, \ldots, x_{4}, t\right) \in \mathbb{R}^{5} \mid \sum_{i=1}^{4} x_{i}^{2}-t^{2}=1\right\}$ and $g=e^{2 f(t)} g_{0}$ where $f(t) \equiv \frac{1}{2} \ln \left(t^{2}+1\right)$ and $g_{0}=R(t)^{2} g_{s^{3}}-d t \otimes d t$ with $R(t) \equiv e^{t^{2}}$ and $g_{s^{3}} \equiv \sum_{k=1}^{3} \prod_{j=k+1}^{3} \cos ^{2} \theta_{j} d \theta_{k} \otimes d \theta_{k}$. For a fixed $s \in[0,1)$, the sectional curvature $K\left(\sigma\left\{v, w_{s}\right\}\right)$ with respect to the plane spanned by $v \equiv \partial \theta_{1}$ and $w_{s} \equiv s R(t)^{-1} \partial \theta_{3}+\partial t$ can be computed to be

$$
\begin{aligned}
K\left(\sigma\left\{v, w_{s}\right\}\right)= & \left(\frac{4 t^{2}}{t^{2}+1}+\frac{2 t^{2}}{\left(t^{2}+1\right)^{2}}\right)-\frac{1}{s^{2}-1}\left(\left[\frac{2}{t^{2}+1}+\frac{1-t^{2}}{\left(t^{2}+1\right)^{3}}\right]\right. \\
& \left.-s^{2}\left[\frac{1}{\left(t^{2}+1\right) e^{2 t^{2}}}+\frac{t^{2}}{\left(t^{2}+1\right)^{3}}+\frac{2 t^{2}}{\left(t^{2}+1\right)^{2}}\right]\right),
\end{aligned}
$$

and hence we have

$$
0<K_{1}(s) \leq K\left(\sigma\left\{v, w_{s}\right\}\right) \leq K_{2}(s)<\infty,
$$

for some positive numbers $K_{1}(s)$ and $K_{2}(s)$. Since the hyperbolic angle between two causal vectors is preserved along geodesics, the angle between a time-like geodesic with initial condition

$$
X_{0}=\left(1-s^{2}\right)^{-\frac{1}{2}}\left(s \partial \theta_{3}+\partial t\right)
$$


and a null geodesic is fixed; hence the time-like geodesic $\gamma_{X_{0}}$ stays away from the light-cone. For every $X_{0} \in \mathfrak{F} \mathbf{M}$, it is possible to find a system of coordinates around $\pi\left(X_{0}\right)$ such that $X_{0}$ takes the form (4.40). Hence the condition (4.1) of theorem 4.1 is satisfied for every $X_{0} \in \mathfrak{F} M$. The fact that $K(\sigma)$ is unbounded follows then from the explicit form of $K\left(\sigma\left\{v, w_{s}\right\}\right)$ as $s$ approaches 1 .

Remark 4.8. A straightforward modification of the above example shows that there exists space-time $(\mathbf{M}, g)$ of $\operatorname{dim} \mathbf{M}=2$ with bounded but non-constant curvature.

When the assumptions of theorem 4.1 are satisfied, the stable and unstable fields are space-like Jacobi fields over geodesic curves, which contract (resp. expend) exponentially as $t$ tends to infinity. The vector bundle $T^{c}(\mathfrak{F} \mathbf{M})$ over $\mathfrak{F} \mathbf{M}$ is thus Riemannian and the following result carries over from the purely Riemannian case (cf. [1], [2], [6], [12]).

THEOREM 4.9. If the time-like sectional curvature of $(\mathbf{M}, g)$ is positive and uniformly bounded on $\mathbf{M}$, then for arbitrary $X_{0} \in \mathfrak{F} \mathbf{M}$, there exist two $\phi$-invariant immersions;

$$
\mathbf{f}_{X_{0}}^{k}: \mathbf{V}_{X_{0}}^{k} \rightarrow \mathfrak{J} \mathbf{M} \quad(k=s, u),
$$

that are respectively tangent to the stable $($ for $k=s)$ and the unstable (for $k=u$ ) fibres, i.e.

$$
\begin{gathered}
\mathbf{V}_{X_{0}}^{k}=T_{X_{0}}\left(\mathbf{f}_{X_{0}}^{k}\left[\mathbf{V}_{X_{0}}^{k}\right]\right) \\
\phi_{t} \circ \mathbf{f}_{X_{0}}^{k}=\mathbf{f}_{\phi_{t} X_{0}}^{k} .
\end{gathered}
$$

Moreover the image of the immersions

$$
\mathbf{F}_{X_{0}}^{i}:(t, \eta) \in \mathbf{Z}_{X_{0}}^{1} \oplus \mathbf{V}_{X_{0}}^{k} \mapsto \phi_{t} \circ \mathbf{f}_{X_{0}}^{k}(\eta) \in \mathfrak{S} \mathbf{M}
$$

are respectively the stable (for $k=s$ ) and unstable (for $k=u$ ) manifolds of $\mathfrak{\Im} \mathbf{M}$.

Proof. We can, without loss of generality, restrict ourselves to the stable case. For $X_{0} \in \mathcal{M} \equiv \mathfrak{F} \mathbf{M}$, we have an immersion $\tilde{\gamma}: t \in \mathbb{R} \mapsto \phi_{t} X_{0} \equiv X_{0 t} \in \mathcal{M}$ which is the geodesic line on $\mathcal{M}$ determined by $\tilde{\gamma}(0)=X_{0}$ and $\dot{\gamma}(0)=\tilde{\xi}_{X_{0}}$. Since $T_{X_{0}}^{1} \mathcal{M}=\tilde{\gamma}_{*}\left(T_{0} \mathbb{R}\right)$ and $T_{X_{0}}^{c} \mathcal{M}$ are $\mathscr{G}$-orthogonal, we can construct the normal bundle $\mu: V \rightarrow \mathbb{R}$ over $\mathbb{R}$ associated with $\tilde{\gamma}: \mathbb{R} \rightarrow \mathfrak{F} \mathbf{M}$ which is the pull-back of $T^{c} \mathcal{M}$ such that for each $t \in \mathbb{R}$,

$$
\mu^{-1}(t)=V_{t} \equiv T_{X_{0 t}}^{c} \mathcal{M}
$$

Hence, we have the following commutative diagram:

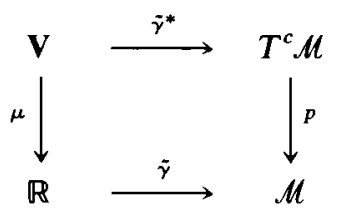

where, for $t \in \mathbb{R},\left.\quad \tilde{\gamma}^{*}\right|_{V_{t}}: V_{t} \mapsto T_{X_{0 t}}^{c} \mathcal{M}$ is a fibre isomorphism. Then $T \phi_{t}$ on $T^{c}(\mathcal{M})$ induces a 1-parameter group of fibre-preserving diffeomorphisms $\left\{\left.\tilde{\phi}_{t} \equiv \tilde{\gamma}^{*-1} \circ T \phi_{t}\right|_{T_{x_{0}}^{c} \mu^{\mu}} \circ \tilde{\gamma}^{*} \mid t \in \mathbb{R}\right\}$ on $\mathbf{V}$ which are symplectic linear transformations on fibres, and the induced bundle $\mu: V \rightarrow \mathbb{R}$ inherits a $\tilde{\phi}_{t}$-invariant splitting $V_{t}=$ $\mathbf{V}_{t}^{s} \oplus \mathbf{V}_{t}^{u}$ for $t \in \mathbb{R}$ with Riemannian metric $\overline{\mathscr{G}}$ on $\mathbf{V} \rightarrow \mathbb{R}$ defined by, for $t \in \mathbb{R}$, $\left.\overline{\mathscr{G}}_{t} \equiv \tilde{\gamma}^{*} \mathscr{G}_{X_{01}}\right|_{T_{X_{0}}^{c}, \mathcal{M}}$ induced by the Lorentz metric $\mathscr{G}$ on $\mathcal{M}$. Since $\tilde{\xi}_{X_{t}}=\hat{\gamma}(t)$ is a parallel 
vector field along $\tilde{\gamma}(t)$ and any parallel translation along $\tilde{\gamma}$ preserves the metric, we obtain a local trivialization of $\mathbf{V}$ with $M^{m+1} \equiv \mathbb{R} \times \mathbb{R}^{m}$, where $M^{m+1}$ is the Minkowski space of dimension $m+1 \equiv 2(n-1)+1$, which is the model space of $\mathcal{M} \equiv \Im$ M. Using this trivialization and the exponential map of $T \mathcal{M}$ over $\mathcal{M}$, we obtain the Fermi charts along $\tilde{\gamma}$ on $\mathcal{M}$. Notice that the tangential of the trivialization gives a linear isomorphism of $T_{(t, 0)} \mathbf{V}$ with $T_{\tilde{\gamma}(t)} \mathcal{M}$. In these Fermi charts, the linear parts of the covariant derivatives of vector fields along $\tilde{\gamma}$ are the ordinary derivatives.

Now a sufficiently small tubular neighbourhood of the 0 -section in $\mu: V \rightarrow \mathbb{R}$ is immersed into $\mathcal{M}$ via the Fermi charts. Thus $\mathbf{V}$ gives us a linearization of $\mathcal{M}$; i.e. we have a linear approximation of the flow line $\phi_{t} X$ near the orbit $\phi_{t} X_{0}$ by

$$
t \in \mathbb{P} \mapsto\left(t, \tilde{\phi}_{t} \zeta^{s}, \tilde{\phi}_{t} \zeta^{u}\right) \in \mathbb{R} \oplus \mathbf{V}_{t}^{s} \oplus \mathbf{V}_{t}^{u},
$$

for $\zeta=\left(\zeta^{s}, \zeta^{u}\right) \in \mathbf{V}_{0}^{s} \oplus \mathbf{V}_{0}^{u}$, where $\zeta$ is a vector in the tubular neighbourhood. We can thus write the vector field in $\mathbf{V}$, given by the linear approximation $\tilde{\phi}_{t}$ of the flow, in the form

$$
\left(1, S_{t}^{s} \zeta^{s}, S_{t}^{u} \zeta^{u}\right) \in T_{(t, \zeta)} \mathbf{V},
$$

where $S_{t}^{s}$ and $S_{t}^{u}$ are the operators obtained by the restrictions of $\tilde{\phi}_{t}$ on $\mathbf{V}^{s}$ and $\mathbf{V}^{u}$ respectively. This vector field can be chosen to be time-like by having $\zeta$ sufficiently small.

Upon considering the time-like geodesic vector field $\xi_{H}$ as a perturbation of this vector field, we can rewrite the coordinates of $\xi_{H}$ in the form

$$
\left(1+\kappa^{1}(t, \zeta), S_{t}^{s} \zeta^{s}+\kappa^{s}(t, \zeta), S_{t}^{u} \zeta^{u}+\kappa^{u}(t, \zeta)\right) \in T_{(t, \zeta)} \mathbf{V},
$$

where $\zeta \in \mathbf{V}$ and $\kappa^{*}(t, \zeta)=\mathcal{O}(\|\zeta\|)$ for $* \in\{1, s, u\}$.

In view of the inequalities (4.36) and (4.37), the following equations, for $\zeta_{t}=$ $\left(\zeta_{t}^{s}, \zeta_{t}^{u}\right) \in \mathbf{V}_{t}^{s} \oplus \mathbf{V}_{t}^{u}=\mathbf{V}_{t}$, are well-defined; for $t \geq 0$, and for each $\zeta^{s} \in \mathbf{V}_{0}^{s}$ in the tubular neighbourhood,

$$
\begin{aligned}
& \zeta_{t}^{s} \equiv \zeta^{s}\left(t, \zeta^{s}\right)=\tilde{\phi}_{t} \cdot \zeta^{s}+\tilde{\phi}_{t} \int_{0}^{t} \tilde{\phi}_{-\tau} \cdot \kappa^{s}\left(\tau, \zeta_{\tau}\right) d \tau \\
& \zeta_{t}^{u} \equiv \zeta^{u}\left(t, \zeta^{s}\right)=\tilde{\phi}_{t} \int_{\infty}^{t} \tilde{\phi}_{-\tau} \cdot \kappa^{u}\left(\tau, \zeta_{\tau}\right) d \tau
\end{aligned}
$$

have a solution $\zeta_{t} \equiv \zeta\left(t, \zeta^{s}\right)$ depending differentiably on $\zeta^{s}$ (cf. [1], [12]).

Then, from (4.36) and (4.37), we have

$$
\left\|\zeta_{t}\right\| \leq 2 \frac{K_{2}}{K_{1}}\left\|\zeta^{s}\right\| e^{-c t}
$$

where $c$ is a constant such that $0<c<K_{1}<2 c$. Hence, we can write

$$
\tilde{\zeta}_{t} \equiv \tilde{\zeta}\left(t, \zeta^{s}\right)=\left(\zeta_{t}^{1}, \zeta_{t}^{s}, \zeta_{t}^{u}\right) \in \mathbb{R} \oplus \mathbf{V}_{t}^{s} \oplus \mathbf{V}_{t}^{u},
$$

where $\zeta_{t}^{1}=\int_{\infty}^{t} \kappa^{1}\left(\tau, \zeta_{\tau}\right) d \tau$. Then the tangent vector of the curve $t \mapsto(t, 0,0)+\tilde{\zeta}_{t}$ is precisely the vector $(4.49)$ at $\left(t, \zeta_{t}\right) \in V_{t}$, i.e. $(t, 0,0)+\tilde{\zeta}_{t}$ is the linear representation of $\phi_{t} X$ near $\phi_{t} X_{0}$.

We now define $f_{X_{0}}^{s}$ on a neighbourhood of the origin in $T_{X_{0}}^{c}, \mathcal{M}$ :

$$
\mathbf{f}_{X_{0}}^{s}: \zeta^{s} \in \mathbf{V}_{0}^{s} \mapsto \mathbf{f}_{X_{0}}^{s}\left(\zeta^{s}\right) \in \mathbb{R} \oplus \mathbf{V}_{t}^{s} \oplus \mathbf{V}_{t}^{u}
$$


with

$$
\mathbf{f}_{X_{0}}^{s}\left(\zeta^{s}\right) \equiv \tilde{\zeta}\left(0, \zeta^{s}\right)=\left(\int_{\infty}^{0} \kappa^{1}\left(\tau, \zeta_{\tau}\right) d \tau, \zeta^{s}, \int_{\infty}^{0} \tilde{\phi}_{-\tau} \kappa\left(\tau, \zeta_{\tau}\right) d \tau\right)
$$

Then $\tilde{\zeta}_{t} \rightarrow 0$ as $t \rightarrow \infty$, i.e. the orbit $\phi_{t} X$ approaches the orbit $\phi_{t} X_{0}$ as $t \rightarrow \infty$. Hence $\phi_{t}$ carries $\mathbf{f}_{X_{0}}^{s}\left(\mathbf{V}_{0}^{s}\right)$ into $\mathbf{V}_{\phi_{t} X_{0}}^{s}$ and for $X \equiv \mathbf{f}_{X_{0}}^{s}\left(\zeta^{s}\right)$, with $\zeta^{s} \in \mathbf{V}_{0}^{s}$ and $\left\|\zeta^{s}\right\|$ sufficiently small, $\mathbf{f}_{X}^{s}$ near $0 \in \mathbf{V}_{X}^{s}$ and $\mathbf{f}_{X_{0}}^{s}$ near $\zeta^{s} \in \mathbf{V}_{X_{0}}^{s}$ have the same image. Now we can extend $\mathbf{f}_{X_{0}}^{s}$ uniquely to an immersion to all of $\mathbf{V}_{X_{0}}^{s}$ since $V_{0}^{s} \cong \mathbb{R}^{n-1}$.

Remark 4.10. From this theorem we conclude that for $X \in \mathbf{f}_{X_{0}}^{s}(\zeta)$ with $\zeta \in \mathbf{V}_{X_{0}}^{s}$, the orbit $\left\{\phi_{t} X \mid t \in \mathbb{R}\right\}$ is asymptotic to $\phi_{t} X_{0}$ as $t$ tends to $+\infty$, i.e. there exists $a \geq 1$ and $b>0$, constant over $\left\{\phi_{t} X \mid t \in \mathbb{R}\right\}$, such that

$$
d\left(\phi_{t} X, \phi_{t} X_{0}\right) \leq a e^{-b t} \quad \text { for } t \geq 0,
$$

where $d$ is the distance function on $\mathfrak{F} \mathbf{M}$ induced from any positive definite bilinear form which is an extension of $\left.\mathscr{G}\right|_{T^{c}(\mathfrak{M})}$ to $T(\mathfrak{I} \mathbf{M})$. The analogous conclusion holds for $Y \in \mathbf{f}_{X_{0}}^{u}(\eta)$ with $\eta \in \mathbf{V}_{X_{0}}^{u}$, as $t$ tends to $-\infty$.

Acknowledgements. The authors would like to thank Christian Günther and Michael Gage for several discussions; and an anonymous referee whose queries prompted Theorem 2.8 as well as the last paragraph of $\S 1$. The first-named author wants to record his gratitude to Prof. H. J. Borchers at the Institut für Theoretische Physik des Universität Göttingen for his hospitality, and to the Akademie der Wissenshaften zu Göttingen for their financial support.

\section{REFERENCES}

[1] D. V. Anosov. Geodesic flows on closed Riemannian manifolds with negative curvature. Proc. Steklov Inst. Math. 90 (1967), 1-235, AMS (1969).

[2] D. V. Anosov \& Ya. G. Sinai. Some smooth ergodic systems. Russian Math. Surveys 22 (1967), 103-167.

[3] V. I. Arnold \& A. Avez. Ergodic Problems of Classical Mechanics. W. A. Benjamin, Inc., New York and Amsterdam, 1968.

[4] R. W. Bass \& L. Witten. Remarks on cosmological models. Rev. Mod. Phys. 29 (1957), 452-453.

[5] J. K. Beem \& P. E. Ehrlich. Global Lorentzian Geometry. Marcel Dekker, Inc., New York and Basel, 1981.

[6] P. Foulon. Contribution a l'étude géometrique des problèmes de la dynamique lagrangienne, \& Géometrie des equations differentielles du second ordre. Preprints, Ecole Polytechnique, Paris, 1983 \& 1984

[7] M. Golubitsky \& V. Guillemin. Stable Mappings and their Singularities. Springer-Verlag, New York, 1980.

[8] J. Hadamard. Les surfaces à courbures opposées et leurs lignes géodésiques. J. Math. Pures et Appliquées (5) 4 (1898), 27-74.

[9] S. G. Harris. A triangle comparison theorem for Lorentz manifolds. Indiana Univ. Math. J. 31 (1982), 289-308.

[10] S. W. Hawking \& G. F. R. Ellis. The Large Scale Structure of Space-Time. Cambridge University Press, Cambridge, 1973.

[11] M. W. Hirsch \& C. C. Pugh. Stable manifolds and hyperbolic sets. In Global Analysis, Proc. Symp. in Pure Math. (AMS) 16 (1970), 133-163.

[12] W. Klingenberg. Riemannian Geometry. Walter de Gruyter, New York, 1982.

[13] E. H. Kronheimer \& R. Penrose. On the structure of causal spaces. Proc. Camb. Phil. Soc. 63 (1967), 481-501. 
[14] D. Lerner. The space of Lorentz metrics. Comm. Math. Phys. 32 (1973), 19-38.

[15] C. Lockhart, B. Misra \& I. Prigogine. Geodesic instability and internal time in relativistic cosmology. Phys. Rev. D25 (1982), 921-929.

[16] B. O'Neill. Semi-Riemannian Geometry. Academic Press, New York, 1983.

[17] A. G. Walker. Completely symmetric spaces. J. Lond. Math. Soc. 19 (1944), 219-226.

[18] J. Wolf. Spaces of Constant Curvature. McGraw Hill, New York, 1967. 